bro-spinal meningitis; embolism, for the history of the case furnishes nothing to indicate the existence of any of these affections.

In some instances it is not an easy matter to distinguish between hæmophilia and morbus maculosus. Our present case, however, does not present the hereditary element which is an essential of the former disease, nor did the patient furnish any evidence of an hereditary hæmorrhagic tendency. Nothing in his record would suggest this unless, possibly, the fact that his gums were apt to bleed slightly when he made use of a stiff tooth-brush, a not uncommon occurrence with many of us.

Under some exceptional circumstances it is difficult to distinguish between scurvy and morbus maculosus. Our patient, however, was in good circumstances, hygienic and otherwise; had been accustomed to the ordinary variety of diet; had been subject to no privation. No similar case occurred in the family or neighborhood. The disease evidently was "spontaneous" in its origin. There were no distinct prodromic symptoms, except a feeling of languor and inability to attend to his ordinary duties for a few days. Previous to that time he was in perfect health. The gums, from which the blood was seen to exude, presented a perfectly healthy appearance. As far as we were able to judge, none of the local symptoms afforded any evidence of an inflammatory process which is very common in scurvy.

The so-called rheumatic symptoms presented by our patient, that is, the swollen and painful joints, accompanied by a slight rise of the pulse and temperature, claim, perhaps, further consideration in making the diagnosis. It has already been stated that recent authorities are inclined to agree that mild, apparently rheumatic, symptoms occurring in association with phenomena which, without them, would indicate the existence of the purpuric disease, may, with good reason, be regarded as symptomatic merely. Reference to the recorded experience with acute rheumatism cannot but confirm this view. It has been shown that with the association of the symptoms the rheumatic elements never obtain any decided prominence; that they are not attended by notable perspiration and never are followed by the grave complications peculiar to the articular disease. In some undoubted cases of rheumatism extravasations of blood do occur, but it is known that this accident is due to embolic processes which have their origin in endocarditis ulcerosa, a condition of things which the clinical history of our patient could not suggest.

Admitting, however, with many, that it is impossible, with our present knowledge, to decide absolutely whether one or the other class of symptoms is symptomatic, it will be evident from the whole character of this paper that I rely upon the theory of probabilities in these cases in making my diagnosis, and so regard the hæmorrhagic affection as the essential disease.

It occurred to me, and has been suggested by others, that the condition presented by my patient may have been due to poisoning by salicylic acid, he having taken in all about three hundred grains. This supposition, however, may be dismissed with a few words. I know of no case on record where this agent is supposed to have given rise to symptoms resembling those presented by my patient. Excessive and long-continued doses, and smaller amounts in certain individuals, of this acid have been known to occasion a condition of extreme prostration, in some cases sudden and rapid collapse, together with cerebral symptoms varying from those resembling cinchonism to acute delirium. Sometimes, too, it produces a miliary eruption which often becomes pustular in character. Urticaria, also, occasionally occurs as a complication. In no case, however, has the eruption become hæmorrhagic in character. Hæmorrhagic erosions of the stomach and intestines have been observed, but in these cases this accident has been considered to be due, probably, to adulteration with carbolic acid.

GROWTH OF BONE IN KNEE-JOINT DISEASE FROM MEASUREMENTS OF NINETY - TWO CASES.

BY J. J. BERRY, M. D.,

Late Interne of Hospital for Ruptured and Crippled, New York.

The question which has been agitated during the past few years regarding the influence of disease of the joints upon the bones entering into their formation has resulted in the publication of very few cases illustrative of these changes. Jutland ${ }^{1}$ has reported cases of elongation of the femur associated with disease of the hip-joint. Wagstaffe ${ }^{2}$ publishes twentyfive cases, of which the femur was found to be lengthened in fourteen, and shortened in one case only. Helferich ${ }^{8}$ has given measurements of one hundred and forty-one subjects of caries of the long bones, but without special reference to disease of the knee-joint. In eighteen of these there existed an elongation of the femur in three cases only, while in fifteen some shortening was noticed.

The accompanying statistics were obtaiued from measurements of consecutive cases in children which came under my observation at the daily clinic during the past two years. 'The greatest degree of accuracy attainable was secured, and no reference is made to those cases in which there were well-defined evidences of disease, but in which no accurate notes were taken.

Of the cases under consideration sixty-eight were of articular osteitis, and gave the following results :-

$$
\begin{aligned}
& \text { In } 2 \text { cases - . . . } 1 \text { inch lengthening. } \\
& \text { In } 10 \text { cases } \quad . \quad \text {. } \frac{3}{4} \text { inch lengthening. } \\
& \text { In } 19 \text { cases } \quad \text { - . } \quad \frac{1}{\frac{1}{2}} \text { inch lengthening. } \\
& \text { In } 18 \text { cases } \quad \text { - . . } \frac{3}{8} \text { inch lengthening. } \\
& \text { In } 8 \text { cases . . . . } \frac{1}{4} \text { inch lengthening. }
\end{aligned}
$$

In six there was an average shortening of three eighths of an inch, while in five no change in length was appreciable. Owing to the difficulty of estimating so slight a variation as a quarter of an inch I would not claim for this measurement any great degree of reliability. In addition to this evidence of bony proliferation there was noticed in most of these cases a marked lateral expansion at the condylar extremity of the femur.

That variety of articular disease in which the morbid process was situated primarily within the synovial membrane, and may be included under the general term of syuovitis, numbered twenty-four. In these there appeared to be little alteration as regards osseous development ; in fact, there seemed to exist in some cases an arrest in growth rather than an intrinsic enlargement, as indicated by this table:-

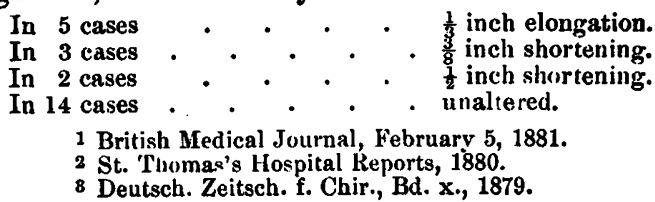


I find among my notes eleven cases of osteitis of the tibia alone, which present the following alterations in size :-

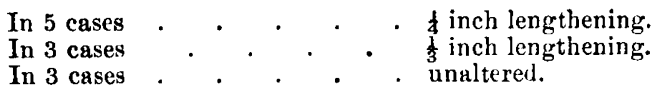

In the above I was unable to recognize any shortening of the tibia. All variations depend upon one of several distinct conditions. The duration of the disease before measurements are taken must be considered. There is absolutely no true bony proliferation after the epiphysis has become united to its shaft, and ordinarily very little during the first six months or after the second year; for the prolonged hyperæmia attendant upon the necrotic process at or near the epiphysal junction advances too sluggishly and furnishes too few products of retrograde change to affect visibly the enveloping structure. It is not until the degenerative process, which we may now term miliary tuberculosis, has encroached upon the larger portion of the epiphysis and its cartilage, separated as it is by its calcareous lamina, and cell proliferation has proceeded unceasingly for many months, that bony deformity begins to manifest itself. I may state in this connection that a case of true osteitis which dates its inception subsequent to the twelfth year of life is of very rare occurrence.

The inconstant normal variation in the length of the femur should also be recognized: for it may serve to explain in a measure the elongation sometimes found in cases of synovitis when not accounted for by the deposition of layers of new bone about the periphery of the articular extremity.

As regards the location of the lesion, no case of femoral osteitis was examined in which the internal condyle was exempt from disease, though in many instances the whole lower third of this bone seemed to share in the inflammation.

The nature of the process is no longer obscure; for the researches of nearly all the modern investigators in this field of inquiry, as imperfectly indicated in a previous paper, ${ }^{1}$ have illustrated quite satisfactorily its tubercular characteristics. They also appeared to have afforded additional weight to the theory that the effect of early synovial inflammation and also some forms of tuberculosis is not an atrophy or even sclerosis of the structure involved, but simply an arrest of development in one portion while the other centres of ossification perform their office uninterruptedly.

\section{RECENT PROGRESS IN PATHOLOGY AND PATHOLOGICAL ANATOMY.}

BY William F. WhitNeY, M. D.

CHRONIC INTERSTITIAL NEPHRITIS AND ITS REI.ATIONS.

THE occurrence of granular atrophy of the kidney, associated with hypertrophy of the heart, has been noticed since the time of Bright. Several hypotheses have been advanced as to the causal relation of the two. Bright himself explained it as a secondary phenomenon, resulting from the changed condition of the blood working on the heart. Traube also considered it as secondary, due, however, to an increase in the arterial 1 Articular Osteitis of the Knee in Children. New York Medical
Record, January 31, 1880 . tension, following the obliteration of the vessels in the kidney, and consequent accumulation of water in the blood. Johnson, Sutton, and Gull have turned their attention to the changes in the arterioles of the entire body, and find in them a thickening of the walls and a reduction in the size of the opening. They are, therefore, led to consider the changes in the vessels as the primary lesion, to which the hypertrophy of the heart and the contraction of the kidney are associated later. Ewald confirms the fact that changes in the vessels occur, but, contrary to the above mentioned authors, considers the affection of the kidney as primary, which gives rise to changes in the blood, leading to increased tension in the capillaries of the entire body.

Still later authors, as Ziegler, recognize this arterial sclerosis as a frequent cause of the contracted kidney. Bartels denies this ætiology entirely, considering the interstitial nephritis as a disease sui generis, while Buhl sees in the fatty and fibroid degeneration of the arteries merely the result of the increased pressure from the hypertrophy of the heart, and not a cause of the interstitial nephritis.

The authors also differ among themselves as to the nature of this thickening of the coats of the vessels. Accordirg to Gull and sutton there is found a layer of a peculiar hyaline, fibroid material upon the muscular coat, and they called this condition arterio-capillary sclerosis. The muscularis itself was unaltered, or else atrophied by pressure of the connective tissue. On the other hand, Johnson found a thickening of the middle coat of the smallest arteries. Dickinson, a thickening of the adventitia and muscularis. Ewald believes in the true hypertrophy of the muscular coat, but finds no abnormal relations in the adventitia or intima.

In order to reconcile these conflicting opinions, which are given more in detail in the original article, Sotnitschewsky, ${ }^{2}$ under the direction of Von Recklinghausen, examined the vessels in seventeen cases of interstitial nephritis. The vessels of the pia mater were the special object of investigation, as they could be examined directly in the tissue without teasing. The small vessels of other organs were not, however, overlooked.

The seventeen cases gave the following result :-

(1.) Thirteen cases had more or less developed genuine contracted kidney and were all accompanied by cardiac hypertrophy. Changes in the small arterioles could be recognized in all the cases but one, which was a case of glomerulitis.

(2.) Three cases had more the character of senile atrophy, although all were accompanied by changes in the vessels. Hypertrophy of the heart occurred in but one case.

(3.) One case showed a well marked old glomerulitis with quite recent round cell infiltration of the connective tissue.

Of the fifteen cases in which hypertrophied heart occurred there were six with no other changes in the circulatory system. In two cases there was valvular disease; while the remaining six showed chronic dis ease (atheroma or sclerosis) of the aorta.

The microscopic examination showed an increase of the adventitia and intima, while the muscularis was only affected secondarily, a fatty degeneration of the nuclei being abserved. Similar changes were seen in the small vessels of the spleen and kidney, less distinctly in the liver and scarcely at all in the lungs.

The limited number of cases makes the author

2 Virchow's Archiv, vol. Ixxxii., p. 212. 